\title{
Transclival Pseudomeningocele Secondary to Ecchordosis Physaliphora: Case Report and Literature Review
}

\author{
Sakshi Kaul ${ }^{1}$ Osaama H. Khan ${ }^{1} \quad$ Idara Edem ${ }^{1}$ Shelley Lwu ${ }^{1}$ Robert Willinsky ${ }^{2}$ Allen Vescan ${ }^{3}$ \\ Gelareh Zadeh ${ }^{1}$ \\ ${ }^{1}$ Division of Neurosurgery, Toronto Western Hospital, Toronto, \\ Ontario, Canada \\ 2 Department of Medical Imaging, Toronto Western Hospital, Toronto, \\ Ontario, Canada \\ ${ }^{3}$ Department of Otolaryngology, Mount Sinai Hospital, Toronto, \\ Ontario, Canada \\ J Neurol Surg Rep 2013;74:92-95.

\begin{abstract}
Address for correspondence Osaama H. Khan, MD, MSc, Division of Neurosurgery, Toronto Western Hospital, 399 Bathurst St, Toronto, Ontario, M5T 2S8, Canada (e-mail: osaama.h.khan@gmail.com).
\end{abstract}
Abstract
Keywords
- ecchordosis physaliphora
- clivus defect
- pseudomeningocele
- meningitis
- transsphenoidal endoscopic surgery

We describe a 52-year-old woman who presented with meningitis secondary to a pseudomeningocele within the sphenoid sinus derived from a bony defect in the clivus. The bony defect was radiologically characteristic of an ecchordosis physaliphora (EP). She underwent surgical repair of the defect and had resolution of her symptoms. This case report will discuss the second case of transclival pseudomeningocele in the English literature and present EP as a cause.

\section{Introduction}

Nontraumatic transclival true pseudomeningoceles are exceedingly rare. They involve the protrusion of an arachnoid sac containing cerebrospinal fluid (CSF) through a bony defect in the clivus. There is only one other reported case in the English literature of a transclival pseudomeningocele. ${ }^{1}$ Similar lesions through the clivus are also rare, and to date there are eight reports in the literature of transclival CSF fistulae and two reports of transclival meningoceles ( $\mathbf{- T a b l e ~ 1 ) . ~ O f ~}$ these cases, only three have demonstrated clear etiologyecchordosis physaliphora $(\mathrm{EP})^{2,3}$ or chordoma. ${ }^{4} \mathrm{EP}$ is a benign notochordal remnant that can present in the intradural space dorsal to the clivus or sacrum. ${ }^{5}$ Although EP is a benign remnant, intradural chordomas are very rare slow-growing tumors arising from EPs that can potentially invade bone. ${ }^{6}$ Radiologically, chordomas would be larger and not cystic in appearance. EPs are not known to invade bone but have been associated with CSF fistulas in only the two cases mentioned above. This case report demonstrates the radiological appearance of an EP, presents a literature review of presentations in cases with known or unknown etiologies of clival defects, and offers a mechanism of how the EP may have produced the rare finding of transclival pseudomeningocele observed.

\section{Case Report}

\section{Clinical History and Presentation}

A 52-year-old right-handed female with a past medical history significant for vertigo, temporomandibular joint disorder, and recurrent otitis media over the past 15 years presented with spontaneous bacterial meningitis approximately 8 months prior to her referral. Meningitis was treated with a 6-weeek course of appropriate intravenous antibiotics tailored to sensitivities from spinal CSF cultures, and she made a full recovery at an outside hospital. One week after received

April 10, 2013

accepted

April 30, 2013

published online

September 9, 2013
DOI http://dx.doi.org/

$10.1055 / \mathrm{s}-0033-1348956$ ISSN 2193-6358. (c) 2013 Georg Thieme Verlag KG
Stuttgart · New York

License terms

(c) $(1) \$$ 
Table 1 Summary of published cases of clivus defect with transclival (pseudo)meningocele or CSF fistula

\begin{tabular}{|c|c|c|c|c|}
\hline Authors (Year) & Age, sex & Type of clival protrusion & Signs and symptoms & Other findings \\
\hline Lockwood et al $1980^{11}$ & $63, F$ & Meningocele into sphenoid & Intermittent CSF rhinorrhea & \\
\hline Macdonald et al $1990^{3}$ & $66, \mathrm{~F}$ & $\begin{array}{l}\text { CSF fistula into sphenoid, } \\
\text { ecchordosis physaliphora }\end{array}$ & $\begin{array}{l}\text { CSF rhinorrhea } \\
\text { (worse w/forward bend) }\end{array}$ & Anosmia \\
\hline Coiteiro et al $1995^{8}$-case 1 & $53, \mathrm{~F}$ & CSF fistula into sphenoid & Meningitis, CSF rhinorrhea & $\begin{array}{l}\text { Basilar artery } \\
\text { observed pulsating } \\
\text { through dural defect }\end{array}$ \\
\hline Coiteiro et al $1995^{8}$-case 2 & $43, M$ & CSF fistula into sphenoid & $\begin{array}{l}\text { Bacterial meningitis, nasal drip, } \\
\text { loculated fluid in sphenoid }\end{array}$ & \\
\hline Schick et al $1998^{10}$ & $55, F$ & CSF fistula into sphenoid & Sinusitis, headache & \\
\hline Ramos et al $2007^{9}$ & $36, M$ & CSF fistula & $\begin{array}{l}\text { Rhinorrhea, occipital headache, } \\
\text { vomiting }\end{array}$ & $\begin{array}{l}\text { Known Marfan } \\
\text { syndrome }\end{array}$ \\
\hline Akyuz et al $2008^{7}$ & $36, F$ & Meningocele into sphenoid & Intermittent CSF rhinorrhea & \\
\hline Alli et al $2008^{2}$ & $52, \mathrm{~F}$ & $\begin{array}{l}\text { CSF fistula into sphenoid, } \\
\text { ecchordosis physaliphora }\end{array}$ & CSF rhinorrhea & \\
\hline Ahmad et al $2008^{1}$-case 1 & $50, M$ & CSF fistula into sphenoid & $\begin{array}{l}\text { Intermittent CSF rhinorrhea } \\
\text { bacterial meningitis }\end{array}$ & \\
\hline Ahmad et al $2008^{1}$-case 2 & $56, M$ & $\begin{array}{l}\text { Pseudomeningocele } \\
\text { into sphenoid }\end{array}$ & $\begin{array}{l}\text { Intermittent CSF rhinorrhea } \\
\text { (worse w/forward bend) }\end{array}$ & $\begin{array}{l}\text { Basilar artery } \\
\text { observed pulsating } \\
\text { through dural defect }\end{array}$ \\
\hline Feng et al $2009^{4}$ & $62, \mathrm{M}$ & $\begin{array}{l}\text { CSF fistula secondary } \\
\text { to clival chordoma }\end{array}$ & CSF rhinorrhea (worse w/URI) & \\
\hline Present study & $52, \mathrm{~F}$ & $\begin{array}{l}\text { Pseudomeningocele } \\
\text { into sphenoid }\end{array}$ & $\begin{array}{l}\text { Meningitis, recurrent otitis } \\
\text { media, tinnitus, ear ache }\end{array}$ & $\begin{array}{l}\text { Basilar artery } \\
\text { observed pulsating } \\
\text { through dural defect }\end{array}$ \\
\hline
\end{tabular}

Abbreviations: CSF, cerebrospinal fluid; URI, upper respiratory infection.

List of published cases of clival defects with transclival pseudomeningocele, meningocele, or CSF fistula.

completing treatment, she developed another ear infection and was referred to an otolaryngologist (AV). With the exception of central and peripheral right vestibular dysfunction, the patient was neurologically intact. There was no CSF rhinorrhea or otorrhea. Imaging investigations were obtained and neurosurgery was subsequently consulted to assist with management.

\section{Investigations}

The noncontrast head computed tomography (CT) scan showed a well-defined and corticated bony defect in the dorsal wall of the clivus, measuring approximately $6 \mathrm{~mm}$, and a soft tissue mass in the sphenoid sinus (-Fig. 1a, b). Magnetic resonance imaging (MRI) revealed a cystic lesion within the bony defect (-Fig. 1c, d). The cyst was isointense to CSF on T1- and T2-weighted images and did not enhance with gadolinium. The cyst was continuous with a fluid loculation that nearly filled the sphenoid sinus. The appearance and location of the cystic lesion was characteristic of EP, and the fluid loculation was in keeping with that of a pseudomeningocele. The tympanic membranes and scutum were intact. The mastoid air cells were clear, and there was no evidence of eustachian tube blockage.

\section{Surgical Management}

Given the history of spontaneous meningitis and the presence of a clival defect with associated pseudomeningocele, there were concerns for CSF leaks and recurrence of meningitis. Hence, the decision was made to proceed with surgical exploration and repair of the clival defect. To assist with localization of the CSF leak intraoperatively, 5 cc of $10 \%$ fluorescein was mixed with $10 \mathrm{cc}$ of CSF and injected intrathecally through a lumbar puncture (LP) and infused slowly for 30 minutes, 3 hours prior to the surgery. Using an endoscopic endonasal approach, the nasopharynx was explored. The sphenoid sinus had no evidence of leakage into the nasal cavity. The sphenoid sinus was entered and drainage of fluorescein-stained CSF through the clival defect was visualized (-Fig. 2a). The sphenoid sinus mucosa was removed and sent for pathological assessment (-Fig. 2b). The underlying 6-mm round defect in the clivus was identified and through it the basilar artery was visible under the arachnoid plane. The bone edges of the clival defect were soft and devoid of any pathology and therefore were not biopsied. There was no associated soft tissue lesions.

A multilayered reconstruction was then performed. The clival defect was repaired with an inlay-onlay Duragen (Integra Neurosciences, Plainsboro, New Jersey, USA) artificial dural substitute (-Fig. $\mathbf{2 c}$ ) and then covered with a vascularized nasoseptal flap (-Fig. 2d). A balloon-inflated Foley catheter was used to buttress the repair. It was left in place for 3 days postoperatively. A lumbar drain was inserted to decrease the CSF pressure on the repair to facilitate healing. 


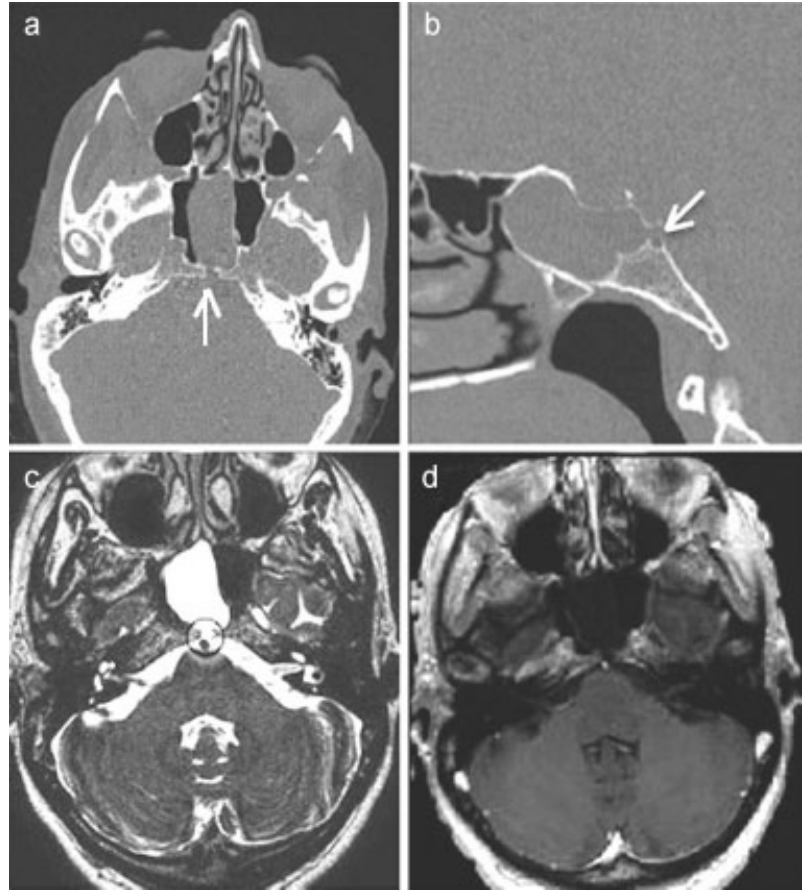

Fig. 1 Radiological imaging. (a) Axial computed tomography (CT) scan revealing defect in clivus bone (arrow). (b) Sagittal CT scan revealing defect in clivus bone (arrow). (c) T2-weighted axial magnetic resonance imaging (MRI) revealing hypointense basilar artery (black circle) directly dorsal to hyperintense cystic lesion characteristic of ecchordosis physaliphora (black circle) and pseudomeningocele in sphenoid sinus. (d) T1-weighted axial MRI with gadolinium enhancement, revealing only enhancement of basilar, and no enhancement of the cystic lesion.

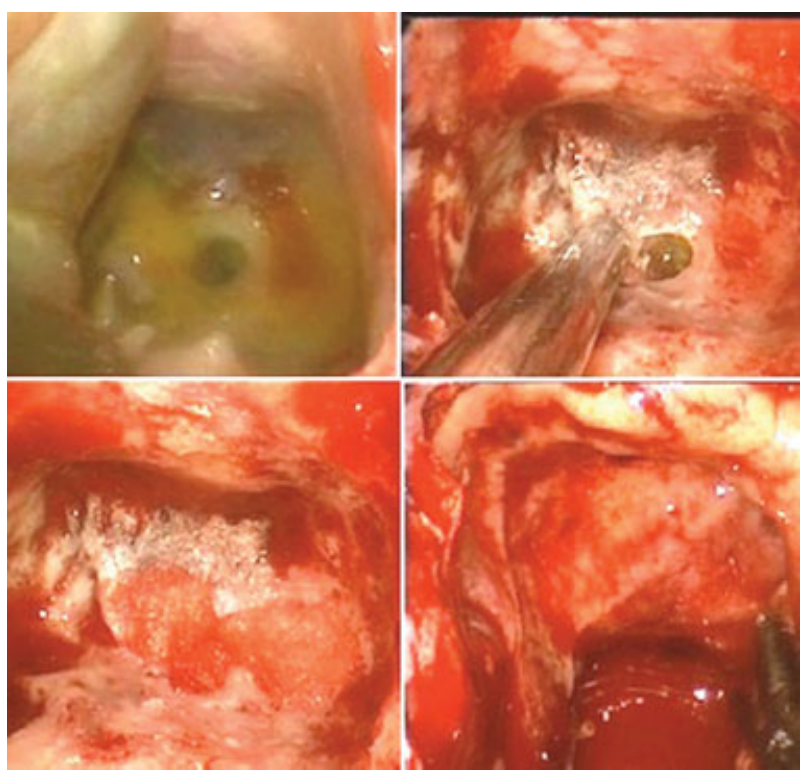

Fig. 2 Intraoperative Images. (a) Clivus defect visible through fluorescein loculation in sphenoid sinus. (b) Clivus defect. (c) Clivus defect repair with inlay-onlay Duragen (Integra Neurosciences, Plainsboro, New Jersey, USA). (d) Placement of septal flap.

A noncontrast head CT done one day after the removal of the lumbar drain revealed scant pneumocephalus with no hydrocephalus. There was still evidence of the presumed EP dorsal to the clival repair. Six weeks postoperatively, the CT scan showed resolution of the pneumocephalus. The patient reported improvement of vertigo and earache. One year postoperatively the patient continued to be well with no reports of meningitis, otitis media, headache, or vertigo.

\section{Pathological Examination}

The wall of the pseudomeningocele was found to contain respiratory mucosa with fragments of bone, dystrophic calcification, and hemosiderin. These findings suggest chronic irritation within the sphenoid sinus.

\section{Discussion}

To date there has been only one other case of pseudomeningocele reported through the clivus. ${ }^{1}$ Although the transclival pseudomeningocele is a rarely reported lesion, similar lesions through the clivus are also rare. There are a total of eight reports in the literature of transclival CSF fistulae and a total of two reports of transclival meningoceles ${ }^{1-4,7-11}$ ( - Table $\mathbf{1}$ ).

A review of clival embryology and anatomy is well beyond the scope of this paper; however, in brief, the clivus is formed by a combination of the basal part of the occipital bone (basiocciput) and the body of the sphenoid bone (basisphenoid) at the spheno-occipital synchondrosis. Postnatal development of the clivus continues till the age of 11 , at which point it reaches its maximal size. ${ }^{12}$ The pathophysiology related to the development of a clival bone defect is not very well defined. It is well appreciated that congenital craniofacial syndromes, such as Alpert syndrome and Crouzon syndrome, are occasionally associated with hypoplasia of the clivus. ${ }^{13}$ There are no clear studies reporting the incidence of spontaneous CSF leak in congenital craniofacial syndromes. Ko had recently published a report of a 7-month old infant with a basiooccipital meningocele that was repaired via endoscope and reviews three other cases previously reported. ${ }^{14}$ All of the cases were associated with a congenital defect in the clivus.

Previous reports of CSF fistulas, meningoceles, and pseudomeningoceles have largely alluded to increased intracranial pressure or normotensive pressure changes being the primary cause of the development of these conditions. Original papers that discussed the role of pressure changes primarily addressed middle fossa bone defects, such as in the lateral sphenoid, ${ }^{15,16}$ cribriform plate, and sellar floor. ${ }^{16}$ In 1979 , Kaufman et $a{ }^{17}$ proposed that fistulae can occur as the result of enlargement of normally present fenestrations in the middle fossa with repeated variations of intracranial pressure and arterial pulsations. Though conceivable that a similar theory could apply to the clivus, it remains to be proven whether in fact gradual dehiscence of the clival bone can occur as a result of the same mechanism given that the clivus in comparison to the cribriform plate, sellar floor, and lateral sphenoid is a much thicker bone. In our patient, for example, the sella floor and lateral sphenoid bones showed no defects. The defect was only within the thick clivus.

In our opinion the occurrence of bony defect in such a location as the clivus would likely need to involve other contributing factors. Macdonald et $\mathrm{al}^{3}$ and Alli et $\mathrm{al}^{2}$ demonstrated transclival CSF fistulas secondary to EP, and Feng et al ${ }^{4}$ 
demonstrated a transclival CSF fistula secondary to a small intradural chordoma. EP is a benign notochordal remnant that may be found in 0.5 to $2 \%$ of autopsies and is typically seen dorsal to the clivus or sacrum. ${ }^{18}$ It varies in size from a few millimeters to $2 \mathrm{~cm}$. Radiologically, EP typically appears as a mass attached to the clivus by a stalk, and it appears hypointense on T1-weighted images, hyperintense on T2-weighted images, and does not enhance with gadolinium. 5,18,19

If not for a congenital defect, it is difficult to imagine why the thick clivus would contain a well-rounded defect. It may be postulated that the development of the hole in the clivus was due to the combined effect of the EP acting as a pressure point, and the mechanisms described by Kaufman, ${ }^{17}$ such as the beating of the basilar artery. In the case of our patient, the basilar artery was visible right underneath the dural defect and in the arachnoid plane. It may also have been possible that the presumed EP remained, blocking the clival defect, while pulsations of the basilar artery upon it also caused dural erosion, which then allowed for arachnoid membrane and CSF to herniate through slowly over time to create the pseudomeningocele. As dystrophic calcification and fragments of bone were apparent on pathology, the pseudomeningocele was likely a source of chronic irritation and the cause of the patient's meningitis. The only other report of a transclival pseudomeningocele was also shown to have the basilar artery pulsating right behind the defect. ${ }^{1}$

\section{Conclusions}

To our knowledge, this is the first reported case of a transclival pseudomeningocele presenting radiological findings of an EP. This report calls onto increasing the awareness of the possibility of the presence of EP when observing transclival protrusions with spontaneous CSF leaks and/or meningitis, to gain more evidence of the correlation of the congenital defect with such symptomatic findings. Finally, endoscopic transsphenoidal repair is a valid surgical approach for treatment of this condition and its associated symptoms.

\section{Conflict of Interest}

None

\section{References}

1 Ahmad FU, Sharma BS, Garg A, Chandra PS. Primary spontaneous CSF rhinorrhea through the clivus: possible etiopathology. J Clin Neurosci 2008;15(11):1304-1308
2 Alli A, Clark M, Mansell NJ. Cerebrospinal fluid rhinorrhea secondary to ecchordosis physaliphora. Skull Base 2008;18(6):395-399

3 Macdonald RL, Cusimano MD, Deck JH, Gullane PJ, Dolan EJ. Cerebrospinal fluid fistula secondary to ecchordosis physaliphora. Neurosurgery 1990;26(3):515-518, discussion 518-519

4 Feng K, Qiuhang Z, Qiuyi Q. Transclival cerebrospinal fluid rhinorrhea as the initial presenting symptom of a tiny intradural chordoma. J Clin Neurosci 2010;17(8):1083-1085

5 Rotondo M, Natale M, Mirone G, Cirillo M, Conforti R, Scuotto A. A rare symptomatic presentation of ecchordosis physaliphora: neuroradiological and surgical management. J Neurol Neurosurg Psychiatry 2007;78(6):647-649

6 Cha ST, Jarrahy R, Yong WH, Eby T, Shahinian HK. A rare symptomatic presentation of ecchordosis physaliphora and unique endoscope-assisted surgical management. Minim Invasive Neurosurg 2002;45(1):36-40

7 Akyuz M, Arslan G, Gurkanlar D, Tuncer R. CSF rhinorrhea from a transclival meningocele: a case report. J Neuroimaging 2008; 18(2):191-193

8 Coiteiro D, Távora L, Antunes JL. Spontaneous cerebrospinal fluid fistula through the clivus: report of two cases. Neurosurgery 1995;37(4):826-828

9 Ramos A, García-Uría J, Ley L, Saucedo G. Transclival cerebrospinal fluid fistula in a patient with Marfan's syndrome. Acta Neurochir (Wien) 2007;149(7):723-725, discussion 725

10 Schick B, Brors D, Goedecke A, Prescher A, Draf W. Detection of an occult transclival cerebrospinal fluid fistula by CT and MRI. Neuroradiology 1998;40(12):797-799

11 Lockwood AH, Quencer RM, Page LK. CSF rhinorrhea from a transclival meningocele demonstrated with metrizamide CT cisternography. Case report. J Neurosurg 1980;53(4):553-555

12 Krmpotić-Nemanić J, Vinter I, Kelovizć Z, Marusić A. Postnatal changes of the clivus. Ann Anat 2005;187(3):277-280

13 Tokumaru AM, Barkovich AJ, Ciricillo SF, Edwards MS. Skull base and calvarial deformities: association with intracranial changes in craniofacial syndromes. AJNR Am J Neuroradiol 1996;17(4): 619-630

14 Ko AL, Gabikian P, Perkins JA, Gruber DP, Avellino AM. Endoscopic repair of a rare basioccipital meningocele associated with recurrent meningitis. J Neurosurg Pediatr 2010;6(2):188-192

15 Morley TP, Wortzman G. The importance of the lateral extensions of the sphenoidal sinus in post-traumatic cerebrospinal rhinorrhoea and meningitis: clinical and radiological aspects. J Neurosurg 1965;22:326-332

16 Ommaya AK, Di Chiro G, Baldwin M, Pennybacker JB. Non-traumatic cerebrospinal fluid rhinorrhoea. J Neurol Neurosurg Psychiatry 1968;31(3):214-225

17 Kaufman B, Yonas H, White RJ, Miller CF II. Acquired middle cranial fossa fistulas: normal pressure and nontraumatic in origin. Neurosurgery 1979;5(4):466-472

18 Mehnert F, Beschorner R, Küker W, Hahn U, Nägele T. Retroclival ecchordosis physaliphora: MR imaging and review of the literature. AJNR Am J Neuroradiol 2004;25(10):1851-1855

19 Toda H, Kondo A, Iwasaki K. Neuroradiological characteristics of ecchordosis physaliphora. Case report and review of the literature. J Neurosurg 1998;89(5):830-834 